\section{Aplicando psicología positiva en educación médica}

\author{
NURIA PEDRALS G. ${ }^{1, \mathrm{a}}$, ATTILIO RIGOTTI R. ${ }^{2, \mathrm{~b}}$, MARCELA BITRAN C..$^{3, \mathrm{c}}$
}

\section{Applying positive psychology to medical education}

Today we are witnessing an exponential growth in positive psychology (PP), a scientific approach to the most significant purpose of human existence: happiness and meaning of life. As a consequence of PP studies, happiness and wellbeing are now accepted as valid topics of research, despite their subjective and supposedly unscientific essence. Experimental evidence indicates that happiness and wellbeing have biological bases and, thus, can be scientifically approached and eventually changed. We believe that we need to learn from this new discipline and apply its discoveries in our profession as medical educators to foster the full development of our students. In this review, we will summarize the major developments and discoveries of PP, and open the debate on the implications of PP offer to medical education.

(Rev Med Chile 2011; 139: 941-949).

Key words: Education, medical; Psychology, educational; Psychology, positive.

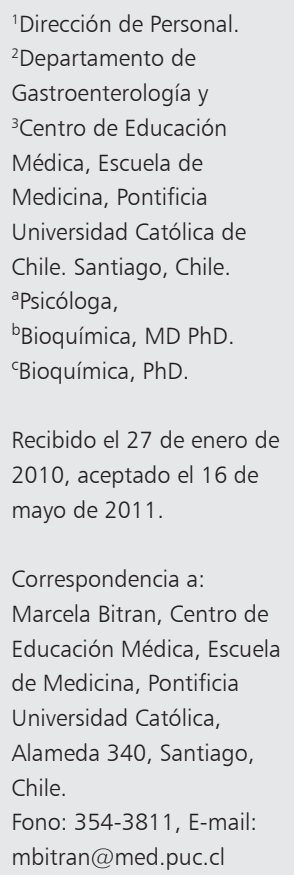

L a prevalencia del estrés crónico y burnout (estado avanzado de estrés profesional caracterizado por agotamiento físico y emocional, despersonalización y baja autoestima) en los profesionales médicos es muy alta ${ }^{1-5}$, particularmente en los residentes de especialidades y subespecialidades ${ }^{6,7}$. Estos estados emocionales negativos afectan desfavorablemente el juicio y la toma de decisiones clínicas ${ }^{5,8-11}$, además menoscaban la satisfacción de los médicos no solamente con su actividad profesional y el clima laboral que los rodea, sino que interfieren con el bienestar y el sentido de sus vidas personales ${ }^{12-14}$. Esta situación no es privativa de la práctica clínica sino que también se manifiesta tempranamente durante los años de pregrado en los alumnos de medicina ${ }^{15-19}$.

De esta realidad emerge la importancia de la evaluación y la implementación de estrategias psicológicas y sociales con sólidas bases científicas durante la formación médica y la práctica clínica que ayuden a los estudiantes y médicos a alcanzar y mantener un mayor nivel de bienestar, satisfacción y sentido vital. En este contexto, la psicología positiva (PP), definida como el estudio científico del origen y las consecuencias del bienestar psicológico y las estrategias necesarias para su desarrollo ${ }^{20}$, en contraste con un enfoque centrado en los estados mentales disfuncionales, ofrece enormes oportunidades. Además, la extensión de estos principios de la PP hacia la relación de los médicos con los pacientes permitiría que éstos sean tratados por profesionales capaces de ayudarlos a potenciar su bienestar y felicidad más allá de la simple búsqueda de la cura de sus enfermedades $^{21-24}$. Esta visión tiene enorme relevancia para la formación del profesionalismo médico ${ }^{25}$, una competencia definida por el conjunto de valores, comportamientos y relaciones que sustentan la confianza que la sociedad tiene en los médicos.

En esta revisión, analizamos el origen, los conceptos básicos y el desarrollo de la PP y sus implicancias para la educación médica.

\section{Origen y principios básicos de la psicología positiva}

Durante la última década, diversos grupos de investigación han aplicado un análisis científicoexperimental al estudio de los aspectos positivos de las personas. Esta nueva área de desarrollo se 
conoce como psicología positiva (PP) y su objetivo es identificar los determinantes individuales y colectivos de las experiencias positivas, y desarrollar intervenciones que permitan a los individuos y las instituciones lograr satisfacción en la vida y alcanzar un mayor potencial de desarrollo ${ }^{20}$. En palabras del Dr. Martín Seligman, el padre de esta nueva rama de la psicología, "la Psicología Positiva se basa en el supuesto que la gente quiere algo más que poner fin a sus sufrimientos. La gente quiere llevar una vida con satisfacción y sentido, cultivar lo que es mejor de ellos mismos y mejorar sus experiencias personales, familiares, de trabajo y en la comunidad. Tenemos la oportunidad de crear una ciencia y una profesión que no sólo cure daños psicológicos, sino que también desarrolle fortalezas para permitir a las personas lograr lo mejor de ellas mismas en la vida". Es decir, la PP busca comprender las causas y las consecuencias del bienestar psicológico en sí mismo, sin considerarlo meramente como la ausencia de estados mentales patológicos. De hecho, el estudio de las bases neurobiológicas del bienestar y de las experiencias positivas ha sugerido que los sustratos anatómicos y neurohormonales corticales de las emociones positivas son diferentes de los que subyacen a las emociones negativas ${ }^{26-28}$.

Esta visión del bienestar personal asociada al estudio neurobiológico y psicológico tradicional es consistente con el modelo biopsicosocial del funcionamiento psíquico humano, el cual aporta una perspectiva integrada de la mente donde cualquier comportamiento o proceso mental afecta y es afectado por factores biológicos, psicológicos y sociales que se interrelacionan dinámicamente. De hecho, el surgimiento de la afectividad mental positiva no constituye un contrasentido biológico, sino que confiere una ventaja evolutiva. La selección adaptativa de procesos mentales ha favorecido tanto el desarrollo paralelo de emociones negativas y positivas ${ }^{29}$. Mientras las emociones negativas (rabia, temor, rechazo) permitieron respuestas específicas y focalizadas para la sobrevida de nuestra especie frente a diferentes tipos de amenzas, la aparición y la persistencia de vías neurales asociadas a las emociones positivas cumplieron un rol clave durante el desarrollo más avanzado del cerebro al permitir ampliar el repertorio de pensamientos y conductas posibles de las especies superiores. En este modelo subyace el concepto que el cerebro, como sustrato material de la mente, expresa una importante plasticidad que permite potenciar los aspectos positivos de la mente humana mediante intervenciones apropiadas ${ }^{30}$.

Desde la perspectiva de la PP, el bienestar individual deriva de tres dominios complementarios de la vida ${ }^{31}$ :

a) La vida placentera o el predominio de las emociones subjetivas positivas del pasado, presente y futuro por sobre el sufrimiento y las emociones negativas,

b) La vida comprometida ("engaged life"), construida sobre la capacidad de reconocer y usar las fortalezas y virtudes propias en balance con los desafíos planteados para alcanzar una verdadera sintonía ("flow") en el trabajo y otro tipo de actividades, incluyendo el uso del tiempo libre, $y$

c) La vida con sentido, que resulta de la sensación de pertenencia, servicio y compromiso con algo más grande que uno mismo.

Para estudiar estos dominios se han desarrollado diferentes instrumentos de evaluación y se han diseñado una serie de intervenciones basadas en evidencias científicas que han demostrado su eficacia para aumentar el placer, el compromiso ('engagement') y el sentido de la vida ${ }^{32}$.

A diferencia de los movimientos de autoayuda que proponen intervenciones para promover el bienestar sin contar con un fundamento científico, la PP se funda sobre sólidas bases teóricas y experimentales y usa estrategias y técnicas propias de las ciencias biomédicas, cognitivas y sociales. Estas herramientas incluyen técnicas de imagenología avanzada para determinar correlatos neurobiológicos de las emociones positivas, estudios longitudinales y protocolos de estudio prospectivos que evalúan la eficacia e impacto de diferentes intervenciones positivas ${ }^{32,33}$.

Las intervenciones basadas en PP corresponden a aquellas actividades intencionales o terapias que buscan el cultivo de los pensamientos, emociones y conductas positivas. Dentro de las técnicas específicas que han mostrado beneficio destacan la resignificación cognitiva, el saboreo y disfrute de logros, la gratitud y la bondad hacia los demás, el perdón, la escritura de experiencias positivas, la actitud proactiva y constructiva frente al logro de otros y la meditación. Algunas de estas intervenciones han producido beneficios más allá del simple desarrollo de emociones positivas: se han asociado a una mejor salud física y menor riesgo 
y sintomatología de depresión clínica. En forma interesante, un meta-análisis de 51 intervenciones de este tipo con 4.266 personas, sin o con depresión, reveló que la aplicación de intervenciones positivas mejora significativamente el bienestar individual y disminuye los síntomas depresivos. El impacto de estas intervenciones depende de la ausencia o presencia de depresión, la autoinclusión en el estudio, la edad de los participantes, así como el formato y la duración de las intervenciones ${ }^{34}$.

El efecto beneficioso de este tipo de enfoque psicológico llega más allá del estado de bienestar subjetivo y parece tener un impacto funcional significativo en el cerebro y la mente. En este sentido, el estudio neurofisiológico de la práctica meditacional de atención focalizada o monitorización abierta ha mostrado resultados promisorios en la identificación de los mecanismos neurales por los cuales esta práctica ejerce sus efectos favorables y la demostración de la plasticidad de los circuitos cerebrales que subyacen en funciones mentales complejas ${ }^{35}$. Varios estudios recientes realizados en individuos con vasta experiencia en meditación basada en atención focalizada se han correlacionado con cambios significativos en el procesamiento atencional así como en la fisiología y estructura del cerebro, incluyendo un aumento en el flujo sanguíneo ${ }^{36} \mathrm{y}$ el espesor de la corteza cerebral ${ }^{37}$. Sin embargo, estos resultados iniciales deben complementarse con nuevos estudios experimentales, especialmente aplicando protocolos longitudinales prospectivos donde los participantes sean randomizados a meditación o un grupo control activo. Otra área importante de investigación futura en este campo es el estudio de las prácticas de meditación que deliberadamente inducen un estado emocional de afecto positivo, empatía, y compasión por los demás, las cuales son frecuentemente consideradas como complementos indispensables para las prácticas meditativas tradicionales.

Por otro lado, la PP ofrece diferentes oportunidades de desarrollo y aplicación en el campo de la psicología clínica ${ }^{38}$, incluyendo entre otras: 1) la clasificación sistemática y práctica de las virtudes, fortalezas y estados positivos de la mente humana (como contraparte del Manual Estadístico y Diagnóstico (DSM) de Enfermedades Mentales); 2) herramientas validadas como entrevistas estructuradas o cuestionarios para el diagnóstico cualitativo y cuantitativo del bienestar individual; 3) la consideración de deficiencias de ciertos rasgos positivos de la personalidad como causa de enfermedades mentales (por ejemplo, un bajo optimismo como factor contribuyente en el origen de la depresión); 4) el sustento que explica parte de la efectividad de la psicoterapia, tanto para el tratamiento psiquiátrico y la potenciación del bienestar personal, y 5) su aplicación como estrategia preventiva para impedir o atenuar la aparición de enfermedades mentales. De hecho, estas implicancias de la PP para la psicología clínica son consistentes y constituyen los elementos básicos, no explícitamente reconocidos, de la práctica rutinaria de la psicología clínica donde gran parte de las intervenciones están dirigidas a expresar las virtudes y potenciar las fortalezas, en vez de reparar las deficiencias, de los pacientes.

En los últimos años, se ha formado una masa crítica de académicos que está liderando la creación de cursos y programas de pre- y postgrado y la organización de reuniones científicas sobre PP. Concomitantemente, ha habido un aumento exponencial de publicaciones científicas, creación de revistas y libros técnicos y de divulgación y de la disponibilidad de fondos para investigación en este campo emergente.

\section{Implicancias de la psicología positiva para la educación}

Las instituciones educacionales son organizaciones donde la PP tiene enormes proyecciones, porque no sólo influyen en los alumnos durante los años de estudio per se, sino que dejan una huella profunda para el futuro, más allá de los logros académicos. En el modelo tradicional, la excelencia de los colegios, institutos de formación profesional y universidades ha sido evaluada exclusivamente mediante exámenes de conocimientos, tasas de graduación, formación académica del profesorado, acceso a fondos concursables, publicaciones académicas y otros parámetros similares. La educación actual orientada hacia la excelencia académica dice a sus estudiantes: "soluciona este problema, arregla esto otro, supera tus debilidades y entonces lograrás el éxito". En contraste, el enfoque educacional basado en las fortalezas personales les dice: "céntrate en tus talentos $y$ fortalezas para corregir tus debilidades". El enfoque de la PP valora la importancia del bienestar subjetivo para el éxito académico y el desarrollo armonioso de los estudiantes, promoviendo la 
implementación de programas educativos que ayuden a los estudiantes a identificar sus propios talentos, desarrollarlos y aplicarlos en un proceso de aprendizaje de excelencia y que favorezcan el desarrrollo de virtudes como autoconciencia, inteligencia emocional, autoeficiencia, resilencia, optimismo y relaciones interpersonales ${ }^{39}$. Los investigadores de prestigiosas universidades como California, Carolina del Norte, Columbia, Harvard, Michigan, Pensilvania y Stanford han demostrado que los estudiantes que desarrollan este conjunto de características positivas, más allá de los aspectos cognitivo-intelectuales, alcanzan una mayor motivación y mejor rendimiento académico y mejor formación integral ${ }^{39-50}$.

Los estudiantes han mostrado gran interés por la PP, como se ilustra en el sorprendente incremento de la demanda por este tipo de cursos en pregrado y postgrado (Positive Psychology Faculty at Universities. http://www.ppc.sas.upenn.edu/ ppfaculty.htm). Un ejemplo señero es el curso de PP dictado por la Universidad de Harvard, que ha contado con casi 900 alumnos inscritos y se ha convertido en el curso de pregrado más popular en la historia de dicha de universidad ${ }^{51}$. Actualmente, más de 100 otras universidades estadounidenses ofrecen cursos similares en pregrado y postgrado.

\section{¿Qué tiene que ofrecer la psicología positiva a la educación médica?}

Según la Organización Mundial de la Salud, la salud implica no sólo la ausencia de enfermedad, sino también el bienestar físico, mental y social de la persona ${ }^{52}$. Sin embargo, en la realidad de la práctica clínica, el médico se relaciona con su paciente casi exclusivamente desde la enfermedad. Este es el mismo paradigma sobre el que se basa la educación médica actual. Aunque los futuros médicos estudian el funcionamiento normal del organismo, el énfasis de su formación está en la enfermedad y su cura. Los currículos, sobrecargados por la necesidad de seguir el ritmo creciente de los descubrimientos científicos ${ }^{53}$ normalmente no suelen incluir instancias o prácticas que enseñen las bases científicas del bienestar del paciente o fomenten el funcionamiento óptimo del estudiante, aquel que se asocia al bienestar subjetivo y la realización personal; es decir, a la salud, en su acepción más amplia.
Por otra parte, los sistemas de evaluación de los aprendizajes están diseñados para detectar las falencias o brechas en conocimientos y destrezas de los estudiantes respecto de criterios estandarizados, sin considerar las diferencias individuales de talentos, valores, vocación o ritmos de aprendizaje. El problema es que este sistema tiene un alto costo para el estudiante. Diversos estudios demuestran que, a lo largo del pregrado, muchos estudiantes de medicina ven debilitada su vocación y capacidad de empatizar con otras personas y sufren crecientes niveles del estrés y burnout ${ }^{15,54}$. Así, algunos estudiantes terminan el pregrado desmotivados y con una percepción menoscabada de sus talentos y potenciales ${ }^{55}$, a pesar de haber iniciado sus estudios con una motivación y vocación claras, y un sentido de valía personal. Esta situación de sobreexigencia y presión es explicada por los propios estudiantes por la gran carga académica y el ambiente competitivo generado, entre otros factores, por la presión por obtener una beca de especialización. En su percepción, esta situación termina por sobrecargarlos y menoscabar su formación profesional y personal, como también sus relaciones interpersonales ${ }^{56}$.

La psicología positiva, disciplina que estudia las bases científicas del bienestar ${ }^{20}$, tiene mucho que aportar a la práctica médica y a la formación de futuros profesionales de la salud ${ }^{57}$. Diversas evidencias experimentales indican que es posible influir positivamente y de forma sostenida sobre el bienestar subjetivo de las personas mediante la realización sistemática de algunas actividades tales como, la práctica del deporte, la gratitud y el cultivo de mindfulness o 'atención plena' ${ }^{32}$.

El concepto de mindfulness define un estado de atención superior que permite mantener -en situaciones cotidianas- una conciencia expandida acerca de uno mismo, el otro y la situación ${ }^{58}$. La práctica de la 'atención plena' es una de las intervenciones cuyo impacto sobre el bienestar personal ha sido más ampliamente documentado en el ámbito de la salud ${ }^{59}$. Por ejemplo, un estudio randomizado controlado, publicado en JAMA, reportó los efectos positivos sobre el bienestar subjetivo y la empatía en un grupo de médicos y residentes, detectados hasta 15 meses después de realizado un programa de 24 horas de práctica de mindfulness ${ }^{60}$. Las intervenciones basadas en la práctica de mindfulness y autocuidado son cada vez más frecuentes en la formación de profesio- 
nales de la salud y se asocian a reducción del burnout, la ansiedad y otros problemas anímicos en estudiantes de medicina, residentes y médicos ${ }^{61,62}$. Así lo demuestra también el estudio de Weiner y colaboradores quienes identificaron el cultivo de las relaciones, la espiritualidad y el autocuidado como las prácticas más importantes que realizan médicos de atención primaria para promover su propio bienestar $^{63}$. Por otra parte, un estudio publicado en 2011 en Medical Education -la revista de mayor impacto en educación médica- indica que el mindfulness no sólo disminuye el estrés, sino que también favorece el desarrollo de actitudes asociadas con el cuidado centrado en el paciente, como la empatía ${ }^{64}$.

Ronald Epstein, académico de la Escuela de Medicina y Odontología de la Universidad of Rochester en EE.UU. de Norteamérica y experto en esta temática, va más allá al sugerir que la mindful practice ('práctica médica consciente') debiera ser una competencia del profesionalismo médico que trascienda los conocimientos teóricos y la experiencia clínica y se exprese como la habilidad de estar presente, manteniendo una actitud atenta, curiosa y con la mente abierta, para obtener conciencia del propio trabajo médico ${ }^{58}$. Esto no es posible sin un entrenamiento previo que genere hábitos para disminuir nuestra reactividad, es decir, hábitos que nos permita prestar atención a las experiencias sin reaccionar inmediatamente a ellas; hábitos que nos ayuden a notar, observar y dejar fluir pensamientos y emociones, aunque sean desagradables, para actuar con conciencia e intención (no en estado de 'piloto automático') y focalizarnos en la experiencia que estamos viviendo, sin prejuicios ni presuposiciones. Concordante con este planteamiento, en un artículo publicado recientemente en JAMA, se pone de relieve la similitud entre el mindfulness y las estrategias de reducción de los prejuicios que se utiliza, como parte del pensamiento crítico médico, al momento de hacer un diagnóstico ${ }^{65}$. Estos autores sugieren que el desarrollo de la atención plena podría ser de utilidad en la reducción de los errores médicos relacionados con la falta de conciencia del médico acerca de sus propios procesos cognitivos y afectivos.

Aunque suele relacionarse con técnicas de meditación y reducción del estrés, los educadores médicos de Rochester conciben el mindfulness como una experiencia humana natural que puede abordarse mediante distintas actividades cognitivas, físicas y espirituales que incluyen el deporte, la actividad física, y el cultivo de la música (http://www.urmc.rochester.edu/education/ $\mathrm{md} / \mathrm{mp} / \mathrm{mindful}$-practice.cfm). Así, ellos han desarrollado un currículum para el cultivo de la práctica consciente y reportaron recientemente un impacto notable y perdurable sobre el estrés, el estado de ánimo, el burnout, la empatía y el bienestar en médicos de atención primaria inscritos en un programa de educación médica continua ${ }^{60}$. A partir del año 2008, un programa semejante de mindful practice forma parte del curriculum de los estudiantes de tercer año de la carrera de Medicina de la Universidad de Rochester y busca promover en ellos las habilidades de observación atenta, curiosidad crítica, flexibilidad informada y presencia consciente ${ }^{66}$. Será muy interesante conocer los resultados de estas prácticas sobre el desarrollo del pensamiento crítico y el aprendizaje, el rendimiento académico, el profesionalismo global y el bienestar y satisfacción vital de los alumnos.

Por otra parte, existen algunas iniciativas para incorporar la educación basada en el fomento de las fortalezas individuales (strengths-based education) en la formación del médico y otros profesionales de la salud ${ }^{67}$. Estas iniciativas buscan promover el bienestar y florecimiento de los estudiantes y la formación de profesionales preparados para promover la salud en su sentido más amplio. Este enfoque promueve el espíritu de descubrimiento, la reflexión crítica y la revisión de prejuicios y estereotipos ${ }^{68}$, lo que redunda en un mayor bienestar y empoderamiento de los estudiantes.

Por parte del docente, el uso de 'strengths-based education' implica un cambio de perspectiva desde un rol directivo a uno cooperativo y de la confianza en el conocimiento experto del instructor clínico a la exploración de las habilidades, conocimientos y recursos del estudiante ${ }^{69}$. En este paradigma, el docente ayuda al estudiante a identificar áreas de aprendizaje y crecimiento y a construir sobre sus fortalezas y habilidades existentes. Esto no libera al docente de la responsabilidad de señalar las áreas problemáticas, pero permite el trabajo en un contexto positivo, de valoración del estudiante como un ser integral.

El cultivo de las fortalezas individuales en la orientación hacia el trabajo también ha demostrado ser beneficioso para los médicos en su quehacer profesional. Un estudio del Program on Physician Well-Being de la Clínica Mayo demostró que la de- 
dicación de los médicos a actividades profesionales que exploten sus fortalezas y virtudes personales y sean significativas para el individuo es un factor determinante del bienestar ${ }^{5}$. De hecho, el ajuste adecuado entre los intereses de los académicos y las horas totales de trabajo dedicadas a la actividad académica (career fit) se está considerando como una medida preventiva del burnout ${ }^{5}$.

En suma, en la actualidad un número pequeño pero creciente de escuelas de medicina está incorporando en su currículum actividades e intervenciones basadas en la psicología positiva, destinadas a reducir el estrés, promover la atención plena y el profesonalismo y apoyar el desarrollo de los estudiantes basado en sus fortalezas personales. Esto es un comienzo promisorio, si aspiramos a formar profesionales de la salud que sean también expertos en promover el bienestar y florecimiento de sus pacientes y estudiantes.

Desconocemos la existencia de iniciativas de este tipo aplicadas en Escuelas de Medicina de nuestro país, aunque hay artículos nacionales que han abordado el profesionalismo médico ${ }^{68-69}$ y otros que se han enfocado en la correlación entre los tipos de personalidades, aspectos psicológicos, estilos cognitivos y formas de aprendizaje con el rendimiento académico de los alumnos ${ }^{71-76}$, estos trabajos no van más allá del mero análisis de los estados emocionales y mentales disfuncionales que afectan sus estudios ${ }^{77-80}$.

A la luz de la evidencia disponible, parece razonable pensar que la incorporación al currículo de los estudios de medicina de actividades dirigidas al cultivo del pensamiento, los sentimientos y las conductas positivas, $\mathrm{y}$ al desarrollo de las fortalezas individuales será beneficiosa tanto para los estudiantes como para los docentes. Estos beneficios estan relacionados con 1) lograr una mayor sintonía y compromiso (engagement) con el estudio y/o trabajo; 2) desarrollar la capacidad para alcanzar un estado de conciencia y atención plena (min$d f u l n e s s$ ) que favorece la empatía, el pensamiento crítico, el aprendizaje y el profesionalismo, y 3 ) aumentar su bienestar subjetivo mejorando el manejo del estrés y previniendo el burnout.

\section{Conclusiones}

Creemos que la PP tiene mucho que aportar a la educación en general, y a la educación médica en particular ${ }^{81}$. Este campo de la psicología puede ser el motor que nos anime como educadores a cambiar el énfasis desde una enseñanza ocupada tradicionalmente de corregir las deficiencias y debilidades de los estudiantes, hacia una práctica docente interesada en identificar sus virtudes y fortalezas, y en nutrirlas a través del aprendizaje experiencial, con actividades de retroalimentación, evaluación y consejería apropiadas.

La adopción de estrategias de la PP en la enseñanza y aprendizaje a lo largo del plan de estudio de las Escuelas de Medicina permitirá a los estudiantes obtener un mejor rendimiento académico y disminuir el riesgo de burnout, estrés y depresión. Más importante aún, aplicar esta aproximación positiva para el desarrollo del potencial humano de nuestros estudiantes de medicina puede ayudarles a desarrollar un trabajo más gratificante durante la etapa de formación y, posteriormente, en su vida profesional y, en útimo término, lograr el objetivo más profundo de toda existencia humana: llevar una vida más feliz y significativa.

Para producir efectos reales, la integración de los conceptos y prácticas de la PP en las Escuelas de Medicina requiere un claro liderazgo de las autoridades y un convencimiento genuino por parte de la comunidad académica que permitan articular un proyecto educativo a largo plazo, contando con participación y apoyo activo de las oficinas de educación médica y los profesores, quienes deben involucrarse plenamente y ser modelos a seguir para sus alumnos.

Es posible que en pocos años más nuestra pregunta inicial ¿qué tiene que ofrecer la $\mathrm{PP}$ a la educación médica? nos parezca ingenua y que sea impensable que un médico no se haya formado profesionalmente en PP y no practique y enseñe técnicas para reducir el estrés, potenciar el compromiso y sintonía con las actividades de la práctica médica diaria y desarrollar ese estado de presencia consciente y atenta que promueve la empatía, el aprendizaje y el pensamiento crítico a lo largo de una vida profesional y personal satisfactoria, plena y con sentido.

\section{Referencias}

1. Campbell DA Jr, Sonnad SS, Eckhauser FE, Campbell KK, Greenfield LJ. Burnout among American surgeons. Surgery 2001; 130: 696-702.

2. Spickard A Jr, Gabbe SG, Christensen JF. Mid-career 
burnout in generalist and specialist physicians. JAMA 2002; 288: 1447-50.

3. Shanafelt TD, Sloan JA, Habermann TM. The well-being of physicians. Am J Med 2003; 114: 513-9.

4. Weinstein L, Wolfe HM. The downward spiral of physician satisfaction: an attempt to avert a crisis within the medical profession. Obstet Gynecol 2007; 109: 1181-3.

5. Shanafelt TD, West CP, Sloan JA, Novotny PJ, Poland GA, Menaker R, et al. Career fit and burnout among academic faculty. Arch Intern Med 2009; 169: 990-5.

6. Thomas NK. Resident burnout. JAMA 2004; 292: 28809.

7. McCray LW, Cronholm PF, Bogner HR, Gallo JJ, Neill RA. Resident physician burnout: is there hope? Fam Med 2008; 40: 626-32.

8. Crane M. Why burned-out doctors get sued more often. Med Econ 1998; 75: 215-8.

9. Fahrenkopf AM, Sectish TC, Barger LK, Sharek PJ, Lewin D, Chiang VW, et al. Rates of medication errors among depressed and burnt out residents: prospective cohort study. BMJ 2008; 336: 488-91.

10. West CP, Tan AD, Habermann TM, Sloan JA, Shanafelt TD. Association of resident fatigue and distress with perceived medical errors. JAMA 2009; 302: 1294-300.

11. Shanafelt TD, Balch CM, Bechamps G, Russell T, Dyrbye L, Satele D, et al. Burnout and medical errors among American surgeons. Ann Surg 2010; 251: 995-1000.

12. Williams B. Pursuing their passions. When they're not seeing patients, these doctors are living out their nonmedical dreams. Tenn Med 2007; 100: 28-35.

13. Carrese JA, Ibrahim MA. Success, regret, and the struggle for balance. Ann Fam Med 2008; 6: 171-2.

14. Foster C. Why doctors should get a life. J R Soc Med 2009; 102: 519-20.

15. Dyrbye LN, Thomas MR, Huschka MM, Lawson KL, Novotny PJ, Sloan JA, et al. A multicenter study of burnout, depression, and quality of life in minority and nonminority US medical students. Mayo Clin Proc 2006; 81: 1435-42.

16. Dahlin ME, Runeson B. Burnout and psychiatric morbidity among medical students entering clinical training: a three year prospective questionnaire and interviewbased study. BMC Med Educ 2007; 7: 6.

17. Dyrbye LN, Thomas MR, Massie FS, Power DV, Eacker A, Harper W, et al. Burnout and suicidal ideation among U.S. medical students. Ann Intern Med 2008; 149: 33441.

18. Dyrbye LN, Thomas MR, Harper W, Massie FS Jr, Power DV, Eacker A, et al. The learning environment and medical student burnout: a multicentre study. Med Educ 2009; 43: 274-82.
19. Jennings ML. Medical student burnout: Interdisciplinary exploration and analysis. Ann Surg 2009; 30: 253-69.

20. Seligman ME, Csikszentmihalyi M. Positive psychology. An introduction. Am Psychol 2000; 55: 5-14.

21. Haas JS, Cook EF, Puopolo AL, Burstin HR, Cleary PD, Brennan TA. Is the professional satisfaction of general internists associated with patient satisfaction? J Gen Intern Med 2000; 15: 122-8.

22. DeVoe J, Fryer Jr GE, Hargraves JL, Phillips RL, Green LA. Does career dissatisfaction affect the ability of family physicians to deliver high-quality patient care? J Fam Pract 2002; 51: 223-8.

23. Pagán JA, Balasubramanian L, Pauly MV. Physicians' career satisfaction, quality of care and patients' trust: the role of community uninsurance. Health Econ Policy Law 2007; 2: 347-62.

24. Quinn MA, Wilcox A, Orav EJ, Bates DW, Simon SR. The relationship between perceived practice quality and quality improvement activities and physician practice dissatisfaction, professional isolation, and work-life stress. Med Care 2009; 47: 924-8.

25. Epstein RM, Hundert EM. Defining and assessing professional competence. JAMA 2002; 287: 226-35.

26. Costa T, Rognoni E, Galati D. EEG phase synchronization during emotional response to positive and negative film stimuli. Neurosci Lett 2006; 406: 159-6.

27. Burgdorf J, Panksepp J. The neurobiology of positive emotions. Neurosci Biobehav Rev 2006; 30: 173-87.

28. Sharot T, Riccardi AM, Raio CM, Phelps EA. Neural mechanisms mediating optimism bias. Nature 2007; 450: 102-5.

29. Fredrickson BL. The broaden-and-build theory of positive emotions. Phil Trans R Soc Lond B 2004; 359 : 1367-77.

30. Wallace BA, Shapiro SL. Mental balance and well-being. Am Psychol 2006; 61: 690-701.

31. Seligman MEP. Authentic happiness: Using the new positive psychology to realize your potential for lasting fulfillment. New York: The Free Press, 2002.

32. Seligman MEP, Steen TA, Park N, Peterson C. Positive psychology progress. Empirical validation of interventions. Am Psychol 2005; 60: 410-21.

33. Snyder CR, Lopez SJ (eds.) Handbook of Positive Psychology. New York: Oxford University Press, 2009.

34. Sin NL, Lyubomirsky S. Enhancing well-being and alleviating depressive symptoms with positive psychology interventions: A practice-friendly meta-analysis. J Clin Psychol 2009; 65:467-87.

35. Lutz A, Slagter HA, Dunne JD, Davidson RJ. Attention regulation and monitoring in meditation. Trends Cogn Sci 2008; 12: 163-9. 
36. Wang DJ, Rao H, Korczykowski M, Wintering N, Pluta J, Khalsa DS, et al. Cerebral blood flow changes associated with different meditation practices and perceived depth of meditation. Psychiatry Res 2011; 191: 60-7.

37. Lazar SW, Kerr CE, Wasserman RH, Gray JR, Greve DN, Treadway MT, et al. Meditation experience is associated with increased cortical thickness. Neuroreport 2005; 16: 1893-7.

38. Duckworth AL, Steen TA, Seligman MEP. Positive psychology in clinical practice. Ann Rev Clin Psychol 2005; 1: 629-51.

39. López SJ. Positive psychology and higher education. 2006; acceso vía http://www.scribd.com/doc/18679036/ Positive-Psychology-and-Higher-Education.

40. Reschly, Huebner ES, Appleton J, Antaramian S. Engagement as flourishing: The contribution of positive emotions and coping to adolescents' engagement at school and with learning. Psychol in Schools 2008; 45: 419-31.

41. Huebner ES, Gilman R, Reschly A, Hall R. Positive schools. In SJ López C. Snyder (Eds.) Handbook of Positive Psychology. New York: Oxford University Press, 2009.

42. Duckworth AL, Seligman MEP. Self-discipline outdoes IQ in predicting academic performance of adolescents. Psychol Sci 2005; 16: 939-44.

43. Judge TA, Hurst C. Capitalizing on one's advantages: Role of core self-evaluations. J Appl Psychol 2007; 92: 1212-27.

44. Sangsue J, Vorpe G. Professional and personal influences on school climate in teachers and pupils. Psychol Trav Organ 2004; 10: 341-54.

45. Goddard RD, Hoy WK, Wolkoff-Hoy AW. Collective efficacy beliefs: theoretical developments, empirical evidence, and future directions. Educ Res 2004; 33: 3-13.

46. Hoy WK, Tater CJ, Wolkoff-Hoy AW. Academic optimism of schools: A force for student achievement. Am Educ Res J 2006; 43:425-46.

47. Schreiner LA, Anderson E. Strengths-based advising: A new lens for higher education. NACADA J 2005; 25: 20 9.

48. Clifton DO, Anderson E, Schreiner LA. StrengthsQuest: discover and develop your strengths in academics, career, and beyond. Washington, DC: Gallup, Inc, 2005.

49. Seligman MEP, Ernst RM, Gillham J, Reivich K, Linkins M. Positive education: positive psychology and classroom interventions. Ox Rev Educ 2009; 35: 293-311.

50. Goddard, R. D. Relational networks, social trust, and norms. A social capital perspective on students' chances of academic success. Educ Eval Policy Anal 2003; 25: 59-74.

51. Goldberg C. Harvard's crowded course to happiness. The
Boston Globe. March 10, 2006.

52. World Health Organization: Constitution of the World Health Organization, Geneva Switzerland (1967). http:// www.who.int/governance/eb/constitution/es/index.html

53. Valdivieso V. La enseñanza de la medicina en el siglo 21: fundamentos y desafíos. Ars Médica 2007; 15: 15-31.

54. Dyrbye L, Thomas M, Power D, Durning S, Moutier C, Massie S, Harper W, Eacker A, Szydlo E, Sloan J and Shanafelt T. Burnout and Serious Thoughts of Dropping Out of Medical School: A Multi-Institutional Study. Acad Med 2010; 85: 94-102.

55. Thomas, M. Dyrbye L, Huntington J, Lawson K, Novotny P, Sloan J, and Shanafelt T. How do distress and wellbeing relate to medical student empathy? A multicenter study. J Gen Intern Med 2007; 22: 177-83.

56. Soemantri D, Herrera C, Riquelme A. Measuring the educational environment in health professions studies: A systematic review. Med Teach 2010; 32 (12): 947-52.

57. Slade M. Mental illness and well-being: the central importance of positive psychology and recovery approaches BMC Health Serv Res 2010; 10: 26.

58. Epstein RM. Mindful practice. JAMA 1999; 282: 833-9.

59. Ludwig D and Kabat-Zinn J Mindfulness in Medicine. JAMA 2008; 300 (11): 1350-2.

60. Krasner MS, Epstein RM, Beckman H, Suchman AL, Chapman B, Mooney CJ, et al. Association of an educational program in mindful communication with burnout, empathy, and attitudes among primary care physicians. JAMA 2009; 302: 1284-93.

61. Shapiro SL, Schwartz GE, Bonner G. Effects of mindfulness-based stress reduction on medical and premedical students. J Behav Med 1998; 21 (6): 581-99.

62. Finkelstein C, Brownstein A, Scott C and Lan Y. Anxiety and stress reduction in medical education: an intervention. Medical Education 2007; 41: 258-64.

63. Weiner EL, Swain GR, Wolf B, Gottlieb M. A qualitative study of physicians' own wellness-promotion practices. West J Med 2001; 174: 19-23.

64. Warnecke E, Quinn S, Ogden K, Towle N and Nelson M. A randomised controlled trial of the effects of mindfulness practice on medical student stress levels. Med Educ 2011; 45: 381-8.

65. Sibinga E and Wu A, Clinician Mindfulness and Patient Safety. JAMA 2010; 304 (22): 2532-3.

66. Katz P. Physicians practice putting mind over matter. ACP Internist, Enero, 2008.

67. Cederbaum J, Klusaritz H. Clinical Instruction: Using the Strengths-Based Approach with Nursing Students. J Nurs Ed 2009; 48 (8): 423.

68. Cadell S, Fletcher M, Makkappallil-Knowles E, Caldwell $\mathrm{S}$, Wong $\mathrm{L}$, Bodurtha $\mathrm{D}$, et al. The use of the arts and the 
strengths perspective: The example of a course assignment. Soc Work Educ 2005; 24: 137-46.

69. Cox AL. BSW students favor strengths/empowerment based generalist practice. J Contemp Human Serv 2001; 82: 305-13.

70. Rosselot E. Dimensiones del profesionalismo médico: Proyecciones para el siglo XXI. Rev Med Chile 2006; 134: 657-64.

71. Fasce E, Echeverría M, Matus O, Ortiz L, Palacios S, Soto A. [Professionalism of physicians from the point of view of physicians and students] Rev Med Chile 2009; 137: 746-52.

72. Bitrán $\mathrm{M}$, Zúñiga $\mathrm{D}$, Lafuente $\mathrm{M}$, Viviani $\mathrm{P}$, Mena B. [Psychological types and learning styles of students entering medical school at the Pontificia Universidad Católica de Chile] Rev Med Chile 2003; 131: 1067-78.

73. Bitrán $\mathrm{M}$, Zúñiga $\mathrm{D}$, Lafuente $\mathrm{M}$, Viviani $\mathrm{P}$, Mena B. [Psychological features and cognitive styles of students entering medicine and other careers at the Pontificia Universidad Católica de Chile] Rev Med Chile 2004; 132: 809-15.

74. Bitrán M, Lafuente M, Zúñiga D, Viviani P, Mena B. [The influence of phychological features and learning styles on the academic performance of medical students]. Rev Med Chile 2004; 132: 1127-36.
75. Bitrán $M$, Zúñiga D, Lafuente M, Viviani P, Mena B. [Influence of personality and learning styles in the choice of medical specialty] Rev Med Chile 2005; 133: 1191-9.

76. Zúñiga D, Mena B, Oliva R, Pedrals N, Padilla O, Bitrán $\mathrm{M}$. [Modeling the academic performance of medical students in basic sciences and pre-clinical courses: a longitudinal study] Rev Med Chile 2009; 137: 1291-300.

77. Benítez C, Quintero J, Torres R. [Prevalence of risk for mental disorders among undergraduate medical students at the Medical School of the Catholic University of Chile] Rev Med Chile 2001; 129: 173-8.

78. Maida AM, Vásquez A, Herskovic V, Calderón JL, Jacard $\mathrm{M}$, Pereira A, et al. A report on student abuse during medical training. Med Teach 2003; 25:497-501.

79. Romero MI, Santander J, Hitschfeld MJ, Labbé M, Zamora V. [Illicit and psychotropic drug use among medical students at the Pontificia Universidad Católica de Chile] Rev Med Chile 2009; 137: 459-65.

80. Romero MI, Santander J, Hitschfeld MJ, Labbé M, Zamora V. [Smoking and alcohol drinking among medical students at the Pontificia Universidad Católica de Chile]. Rev Med Chile 2009; 137: 361-8.

81. Pedrals N, Bitrán M Rigotti A. Focusing on Students' Talents and Strengths in Medical Education. Acad Med 2010; 85 (5): 744-5. 